\title{
An Improved Calibration and Uncertainty Analysis Approach Using a Multicriteria Sequential Algorithm for Hydrological Modeling
}

\section{Hongjing Wu}

Memorial University of Newfoundland

Bing Chen ( $\sim$ bchen@mun.ca)

Memorial University of Newfoundland

\section{Xudong Ye}

Memorial University of Newfoundland

Huaicheng Guo

Peking University

Xianyong Meng

China Agricultural University (CAU)

\section{Baiyu Zhang}

Memorial University of Newfoundland

\section{Research Article}

Keywords: Hydrological Modeling, Uncertainty analysis, Modeling calibration, Sequential algorithm, Multicriteria evaluation

Posted Date: April 12th, 2021

DOl: https://doi.org/10.21203/rs.3.rs-396290/v1

License: (c) (1) This work is licensed under a Creative Commons Attribution 4.0 International License. Read Full License

Version of Record: A version of this preprint was published at Scientific Reports on August 20th, 2021. See the published version at https://doi.org/10.1038/s41598-021-96250-6. 


\title{
An improved calibration and uncertainty analysis approach using a multicriteria sequential algorithm for hydrological modeling
}

\author{
Hongjing Wu ${ }^{\mathrm{a}}$, Bing Chen ${ }^{\mathrm{a}, *}$, Xudong Ye ${ }^{\mathrm{a}}$, Huaicheng Guo ${ }^{\mathrm{b}}$, Xianyong Meng ${ }^{\mathrm{c}}$, Baiyu \\ Zhang ${ }^{\text {a }}$
}

${ }^{a}$ Northern Region Persistent Organic Pollution Control (NRPOP) Laboratory, Faculty of Engineering and Applied Science, Memorial University of Newfoundland, St. John's, NL A1B 3X5, Canada.

${ }^{b}$ College of Environmental Science and Engineering, Peking University, No.5 Yiheyuan Road Haidian District, Beijing, 100871, P. R. China.

${ }^{c}$ College of Resources and Environmental Science, China Agricultural University

(CAU), Beijing 100094, China

*Corresponding author: Bing Chen. Tel: +1 (709) 864-8958. Fax: +1 (709) 864-4042.

Email: bchen@mun.ca

\begin{abstract}
Hydrological models are widely used as simplified, conceptual, mathematical representatives for water resource management. The performance of hydrological modeling is usually challenged by model calibration and uncertainty analysis during modeling exercises. In this study, a multicriteria sequential calibration and uncertainty analysis (MS-CUA) method was proposed to improve hydrological modeling efficiency and performance with high reliability. To evaluate the performance, the proposed MS-CUA method was applied to two case studies comparing two traditional methods: sequential uncertainty fitting algorithm (SUFI-2) and generalized likelihood uncertainty estimation (GLUE). The results indicated that the MS-CUA method can quickly locate the highest posterior density (HPD) regions of parameters to improve computational efficiency. It also provided better-calibrated results and more balanced uncertainty analysis results comparing with other traditional methods.
\end{abstract}

Keywords: Hydrological Modeling; Uncertainty analysis; Modeling calibration; Sequential algorithm; Multicriteria evaluation

\section{Introduction}

Hydrological models have been significantly improved over the past several decades and applied to different applications and water resource management, such as land use management $^{1}$, flood prediction $^{2,3}$, sediment transport simulation ${ }^{4}$, and nutrient yield simulation $^{5}$. The complexity of hydrological models has been dramatically increased (e.g., from lumped models to distributed models), especially for the distributed model ${ }^{6}$. The extensive data and computational requirement, along with large numbers of unadjusted distributed or semi-distributed parameters, always become the obstacles for improving hydrological modeling ${ }^{7}$. Different approaches were developed to assess and enhance hydrological modeling performance. They included parameter optimization ${ }^{8,9}$, calibration $^{10,11}$, design space exploration ${ }^{12}$, sensitivity analysis ${ }^{13,14}$, and uncertainty 
analysis $^{15-17}$.

Most conceptual distributed and semi-distributed parameters cannot be easily determined due to the limitations of parameter measurements from physical systems and available data. Moreover, measurement errors and system uncertainties are inevitable. Therefore, model calibration should be applied to adjust parameters within recommended ranges by optimizing observed and simulated data ${ }^{18}$. Calibration is always challenging due to the uncertainties from model input, model structure, parameter, and output ${ }^{19}$. Uncertainty is a subjective factor of people's confidence in the understanding of individuals and communities. Based on personal experiences, assumptions and knowledge, different people will come to different conclusions on how uncertain something is ${ }^{20}$. Imperfect knowledge, especially in hydrological studies, makes the uncertainty inevitable. As many distributed hydrological models are extensively used to support decision-making process of watershed management, it is essential and necessary to perform careful calibration and uncertainties analysis for these models.

For hydrological studies, parameter uncertainty has always been given the most concerns. According to empirical estimations, the uncertainty of model parameters may compromise the reliability and precision of modeling results, such as physical significance, generalized inference, and observed data ${ }^{21,22}$. Moreover, the interactions between the parameters also cause uncertainties. For example, a single-valued parameter set results in a single output for modeling. However, in inverse applications, multiple combinations of different parameter sets can reproduce an observed output value. This non-unique phenomenon is known as "equifinality", which is an inherent property of inverse modeling demonstrating the uncertainties of parameters ${ }^{23}$. For uncertainty reduction purposes, many existed obvious errors of parameters which can generate reasonable outputs need to be removed. Due to a large number of unknown or unmeasurable parameters and errors in the data, parameter uncertainty must be controlled and quantified ${ }^{24}$.

In the past several decades, several methods have been proposed to address parameter uncertainty. The most traditional statistical way is the first-order approximation, but one of the weaknesses is that the correlation between parameters cannot be evaluated ${ }^{25}$. Other methods for estimating the parameter confidence levels include the uniform grid sampling, uniform random sampling, and sequential uncertainty fitting algorithm (SUFI-2) ${ }^{26-28}$. These methods typically require massive computational resources for the high dimensional model. The sampling scheme may fail to search the global optimum among the parameter space if the initial range is not dense enough ${ }^{25,29}$. Monte Carlo- based approaches, such as Markov Chain Monte Carlo (MCMC) simulation ${ }^{30}$ and generalized likelihood uncertainty estimation (GLUE) ${ }^{31}$, are widely used in hydrological modeling for quantifying parameter uncertainties. These methods can consider the nonlinearity and interdependency of parameters. However, the computational requirement is relatively high when facing complex nonlinear hydrological models (e.g. some complex distributed models) ${ }^{32}$.

The motivation of this study is to propose a method to improve efficiency and accuracy of calibration and uncertainty analysis for supporting hydrological modeling. Therefore, the multicriteria sequential calibration and uncertainty analysis (MS-CUA) method was 
proposed in this study. The feasibility and performance of MS-CUA were evaluated using a hypothetical case provided by SWAT calibration and uncertainty program (SWAT-CUP) and a real case study at the upstream of the Wenjing River watershed in Chongzhou, China. The calibration and uncertainty analysis results were compared with the results of GLUE and SUFI-2. The results indicate that the proposed MS-CUA method can achieve higher efficiency for parameter calibration and more balanced uncertainty analysis results than the other two methods.

\section{Results \& Discussion}

Two case studies were applied to evaluate the performance and efficiency of the proposed methods. The MS-CUA method was applied to simulation results for both cases to test the feasibility and flexibility. A hypothetical case was conducted with demo data from the SWAT Calibration and Uncertainty Programs (SWAT-CUP) (Case 1). The proposed method was simplified by using only two iterations without adding any additional criterion, and the results were compared with GLUE. Moreover, the MS-CUA was also applied to a comprehensive real-world case of the Wenjing River watershed upstream in Chongzhou, China (Case 2). The case study conducted four iterations with the comparison of the results from SUFI-2 and GLUE. The results from the two cases were comprehensively evaluated and analyzed from a simplified and conceptual point to a complicated and practical aspect.

\section{- Case 1: A hypothetical case using the demo data from SWAT-CUP}

The simplified MS-CUA method was applied to a hypothetical case as a preliminary test using the demo data from SWAT-CUP. The NSE was used as the likelihood function. The performance improvements of calibration were tested using two iterations with 1,000 simulations in each iteration. The threshold value was set to 0.8 due to the high match between the simulated and observed surface runoff. In the study, over $35 \%$ of observation should be included in 95PPU iteratively to achieve acceptable uncertainty analysis results. Ten considered parameters included $\mathrm{CN}_{2}$, ALPHA_BF, GW_DELAY, CH_N2, CH_K2, ALPHA_BNK, SOL_AWC, SOL_K, SOL_BD, and SFTMP. The definition of each parameter has been provided in Table 1.

Table 1. Definitions of selected input parameters in SWAT ${ }^{33}$

The LHS method was used to generate 2,000 parameter sets (for two iterations) from prior uniform distributions of each parameter. After the first iteration, the parameter ranges were adjusted according to the NSE results for multi-iteration performance evaluation. The examples of NSE values verse some parameters for iterations 1 and 2 were shown in Figure 1. The 95PPU of surface runoff was obtained through the first iteration, and the new parameter ranges were updated based on ranking the behavioral parameter sets correspondingly. For example, from the plot for parameter $\mathrm{CN}_{2}$ in Figure 1 (A), it is shown that there is an apparent curvature within the original predefined parameter space, and the NSE value increases with the increase of $\mathrm{CN}_{2}$ value. Therefore, the desirable solutions should locate in the region of larger values of $\mathrm{CN}_{2}$ within the predefined feasible space. Through evaluating the likelihood value of the objective function, some impractical parameter sets were removed from behavioral parameter sets. By using refined behavioral parameter sets, the 95PPU and parameter ranges were updated. As a simplified case, only 
parameter $\mathrm{CN}_{2}$ was adjusted for the second iteration. As shown in Figure 1 (B), the lower bound of parameter $\mathrm{CN}_{2}$ was shifted from -0.10 to -0.01 .

Figure 1 Parameter ranges of selected parameters in the first iteration (A) and second iteration $(\mathrm{B})$

The comparison results between the simplified MS-CUA in 2,000 simulation scenarios and GLUE in 10,000 simulation scenarios were shown in Table 2. In outlet 1, the best simulation of the MS-CUA method and GLUE method reached 0.91 and 0.90 for the value of NSE, respectively. Both methods achieved the same $\mathrm{R}^{2}$ values as 0.91 . The results represented that the calibrated simulations from both methods were very close to the observed surface runoff due to the high NSE and $\mathrm{R}^{2}$ values. The good uncertainty analysis searched for the results that bracketed most of the observed data with the smallest possible uncertainty band, which means the good uncertainty analysis results should have a relatively large $\mathrm{P}$-factor with a relatively small $\mathrm{R}$-factor. Therefore, the greater the ratio of $\mathrm{P} / \mathrm{R}$ was, the better the simulation performance achieved. The MS-CUA method provided a smaller R-factor value as 0.30 than the GLUE method (R-factor $=0.53)$ with the same Pfactor value of 0.38 . The $\mathrm{P} / \mathrm{R}$ ratio of the MS-CUA method in outlet 1 is 1.23 , which is much larger than the ratio value of 0.72 of the GLUE. Although the results for outlet 1 barely showed the improvement of MS-CUA over GLUE for the best calibrated simulation, the ratio of $\mathrm{P}$-factor and $\mathrm{R}$-factor indicated that the MS-CUA method provided a better uncertainty analysis results than GLUE. In outlet 2, the best surface runoff simulation results from the second iteration also indicated that the proposed MS-CUA achieved better results than those from GLUE with 10,000 simulation runs. The NSE of MS-CUA and GLUE were 0.97 and $0.96, \mathrm{R}^{2}$ values of the two methods were 0.98 and 0.97 , respectively. They indicated that MS-CUA had a better calibration performance. The P/R ratio of MSCUA was 2.15, which was much greater than GLUE, 1.34. That represented that MS-CUA had a better uncertainty analysis result.

Table 2 also showed the results of the first iteration with original parameter ranges and the second iteration with the updated parameter range (only using updated parameter ranges of $\mathrm{CN}_{2}$ as an example) with the MS-CUA method. The second iteration improved the NSE and $\mathrm{R}^{2}$ of the best simulation for outlet 1 and outlet 2 . Due to the high quality of simulation results, limited improvement was found for the best simulation results in the second iteration. However, through the simplified MS-CUA method, the number of behavioral simulations dramatically increased with the updated parameter ranges. The percentages of behavioral parameter sets among the total parameter sets were larger than the corresponding percentages in GLUE. It indicated that the MS-CUA method can more accurately capture the highest posterior density (HPD) region than the random Monte Carlo sampling method of GLUE. The reasonable and acceptable results can be achieved at the circumstance of dramatically reducing parameter uncertainties indicating the advantages of the proposed MS-CUA method.

Table 2 The comparison results between the simplified MS-CUA method and GLUE

The above analysis clearly showed that the MS-CUA could provide better calibration 
results and uncertainty analysis results than the traditional GLUE method. At the same time, the MS-CUA method was far more efficient compared with GLUE. Only 2,000 simulation runs were conducted for the MS-CUA method and achieved better calibration, and uncertainty analysis results with the comparison of GLUE in 10,000 simulation runs. Another significant advantage of the MS-CUA method was that the parameter uncertainty was reduced by using updated parameter ranges. Therefore, the MS-CUA method can help to reduce uncertainty effectively. The uncertainty reduction was very meaningful, especially for uncertainty propagation problems.

\section{- Case 2: A real case study at the upstream of Wenjing River watershed}

A real case study was conducted to evaluate the feasibility and performance of proposed MS-CUA methods. The upstream of the Wenjing River watershed was selected as the study area. Figure 2 showed the watershed location and digital elevation model (DEM) of the study area ${ }^{10}$.

Figure 2 Location map of the upstream of the Wenjing River watershed. The map is generated with ArcMap Version 10.5.(Wu and Chen, 2015)

\section{(a) Results of MS-CUA}

The MS-CUA method conducted four iterations with 1,000 simulation runs per iteration. To get a reasonable uncertainty analysis, it was assumed that at least $35 \%$ of observed surface runoff should be included in 95PPU for each iteration. A total of 11 parameters involved in calibration and uncertainty analysis, as well as definitions of each parameter, were provided in Table 1. Two comparable scenarios of simulations with one or two criteria were conducted to investigate the performance.

\section{- Simulations using one criterion}

The statistical summary of simulation results was shown in Table 3. In the simulation results with one criterion, the threshold values of the objective function for four iterations were set as different values $(0.20,0.40,0.60$ and 0.60$)$. Since it was difficult to determine the HPD region for the first iteration, a relatively low threshold of the objective function was set for the preliminary HPD estimation of different parameter ranges. When approaching the HPD to obtain smaller parameter ranges, the objective function threshold can be adjusted for each iteration. A higher threshold would result in a smaller number of behavioral parameters. Thus, the threshold value was set to 0.60 for the fourth iteration to guarantee reasonable parameter ranges for different parameters.

After each iteration, the parameter sets generating 95PPU of surface runoff were used to update parameter ranges for the next iteration. The parameter range values of each iteration were shown in Table 4. From the first to fourth iteration, the number of behavioral parameter sets increased from 86 to 410 , indicating the updated parameter ranges are around the HPD region after four iterations. To further investigate the performance of calibration and uncertainty analysis using the MS-CUA method, the P-factor, R-factor, and $\mathrm{R}^{2}$ and NSE values of the best simulation in each iteration were shown in Table 3. Typically, the smaller uncertainty bands resulted in a smaller coverage of observed runoff data. Therefore, the larger value of $\mathrm{P} / \mathrm{R}$ with acceptable coverage was the desired result. 
Although the P-factor slightly decreased from 0.5 to 0.42 during iterations, the R-factor was dramatically decreased from 0.90 to 0.49 simultaneously. The $\mathrm{P} / \mathrm{R}$ ratio increased from 0.56 to 0.86 , which demonstrated the improvement of uncertainty analysis results. The $\mathrm{R}^{2}$ values of four iterations are pretty constant with a high value (above 0.77 ), showing a good correlation between simulated results and observed surface runoff. The NSE values for four iterations increased from 0.68 to 0.71 , and a better-calibrated simulation was achieved. Through the application of the MS-CUA method with one criterion, the ratio of $P / R, R^{2}$ and NSE all increased during the multiple iterations, indicating the improvement of calibration and uncertainty analysis using the MS-CUA method.

Table 3 The statistic summary of the simulation results

Table 4 Parameter range values of each iteration

\section{- Simulations using two criteria}

To further test the proposed MS-CUA method, the simulation with two criteria was proposed to screen the behavioral parameter sets instead of one criterion. The $\mathrm{R}^{2}$ was used as an additional criterion to further screen parameter sets. These screened parameter sets are called "refined behavioral parameter sets" among the behavioral parameter sets. During each iteration, the results for behavioral parameter sets (screened by the objective function) and refined behavioral parameter sets (screened by the objective function and $\mathrm{R}^{2}$ ) in each iteration were also shown in Table 3. The threshold values of the objective function for four iterations were set as the same for the one criterion case $(0.2,0.4,0.6$, and 0.6$)$. The $\mathrm{R}^{2}$ was used as the additional criterion in each iteration. Due to the good correlation between simulated runoff results and observed surface runoff, the values were set as 0.60 , $0.70,0.75$ and 0.75 for the four iterations.

For the first iteration, the threshold value of the objective function was set to 0.20 . Two situations were analyzed separately, including the results for behavioral parameters and the refined behavioral parameter sets in each iteration. The threshold value of second criterion $\mathrm{R}^{2}$ was set to 0.60 for screening the refined behavioral parameters among the behavioral parameter sets. There are 81 refined behavioral parameter sets among 86 behavioral parameter sets in this iteration. Because only five parameter sets were removed from behavioral parameter sets due to the lower $\mathrm{R}^{2}$ values, $\mathrm{P}$-factor and $\mathrm{R}$-factor are the same for the two situations, which are 0.50 and 0.90 , respectively. The values of $\mathrm{R}^{2}$ and NSE of the best-calibrated simulation are 0.79 and 0.68 , indicating reasonably good simulation results. Because the best-calibrated simulation was not removed when using additional criteria, the $\mathrm{R}^{2}$ and NSE of the best simulation will not change for two situations within an iteration. According to the $95 \mathrm{PPU}$ of the 81 parameter sets (screened by using two criteria), the updated parameter ranges for the second iteration were calculated.

For the second iteration, the threshold value of the objective function increased to 0.40 . The 62 behavioral parameter sets can be found. The $\mathrm{R}^{2}$ and NSE values of the bestcalibrated simulation increased to 0.78 and 0.70 , respectively. When the $\mathrm{R}^{2}$ was set to 0.70 for the second iteration, only 34 behavioral parameter sets were left as refined behavioral parameter sets. Therefore, the P-factor and R-factor were different for the two situations. 
The R-factor of two criteria (0.66) was smaller than that of one criterion (0.71), showing reducing the prediction uncertainty. The same P-factor value of 0.47 indicated that the reducing uncertainty did not affect the model prediction power and still covered the same number of observed data. The results with reduced uncertainty through two criteria were preferred results, and the 95PPU of the 34 refined behavioral parameter sets were used to calculate the parameter ranges for the third iteration.

For the third iteration, the threshold value of NSE was set to 0.60 . There are 74 behavioral parameter sets after screening. The $\mathrm{R}^{2}$ and NSE values of the best-calibrated simulation were 0.78 and 0.73 , respectively. The $\mathrm{R}^{2}$ and NSE values were greater than the results in the second iteration, indicating the improvement of the calibrated results. The additional criterion $\mathrm{R}^{2}$ was increased to 0.75 in this iteration for getting better refined behavioral parameter sets. Within 74 behavioral parameter sets, 41 parameter sets can achieve the value of $\mathrm{R}^{2}$ greater than 0.75 . The $\mathrm{R}$-factor reduced from 0.52 to 0.45 when using two criteria. Even though the P-factor reduced a little (from 0.44 to 0.42 ), the P-factor ratio and $\mathrm{R}$-factor ratio increased from 0.85 to 0.93 , showing the improvement of uncertainty analysis results. The 95PPU values of the 41 refined behavioral parameter sets were used to calculate the parameter ranges for the fourth iteration. The exact values of each parameter range for four iterations were shown in Table 4.

For the last iteration, the threshold values of the objective function and the additional criterion were set the same value as the third iteration. Due to the application of the previous three iterations, the HPD region was estimated for each parameter. Therefore, the number of behavioral parameter sets was increased to 605 , and the number of refined behavioral parameter sets was determined to 527 . The $\mathrm{R}^{2}$ and NSE of the best-calibrated simulation are 0.79 and 0.74 , respectively, which are the best-calibrated simulation among the four iterations. The R-factor decreased to 0.39 , with a P-factor of 0.39 for both situations. The ratios of P-factor and R-factor are 1.00, which were the largest value of four iterations. All those values of different indicators showed that the last iteration achieved the best-calibrated simulation with the most balanced uncertainty analysis results.

The MS-CUA method can effectively calibrate the hydrological model and provide reasonably good uncertainty analysis results through four iterations. The best-calibrated simulation results were improved in each iteration, and more balanced and reliable uncertainty analysis results were provided (larger ratio of $\mathrm{P}$-factor and R-factor). The prediction uncertainty decreased in each iteration represented by the 95PPU of surface runoff. Through the multicriteria screen process, the behavioral parameter sets in each iteration were reduced, indicating the reduction of the equifinality phenomenon, which demonstrates the advantages of the MS-CUA method. Figure 3 showed the 95PPU of surface runoff in each iteration. The 95PPU bands were decreasing during each iteration. The uncertainty bands moved towards the observed runoff and tended to cover more observed results. SUFI-2 and GLUE were applied to the same case for comparison to evaluate the proposed method's performance.

Figure 3 The 95PPU of surface runoff for four iterations using the MS-CUA method 


\section{(b) Comparison results of SUFI-2 and GLUE \\ - SUFI-2}

The calibration and uncertainty analysis by using the SUFI-2 was firstly conducted. The NSE was used as the likelihood function, and the threshold value of the objective function was set to 0.40 . The four iterations with 1,000 simulations in each iteration were conducted. For a better comparison, the initial parameter ranges were set as the same as the parameter ranges for the first iteration of the MS-CUA method. The parameter range settings for each iteration were shown in Table 5. According to the evolution of parameter ranges in SUFI2 , the parameter ranges decreased during different iterations, and parameter ranges were centered on the best estimates ${ }^{10}$. The detailed statistics for each iteration were shown in Table 6. The $\mathrm{R}^{2}$ and NSE of the best-calibrated simulation were 0.78 and 0.75 in the fourth iteration, respectively. It indicated an outstanding calibration result. The best-calibrated simulation results are slightly better than the fourth iteration calibrated results using the MS-CUA method (due to the higher NSE value). In the fourth iteration, the P/R ratio was 0.93, which was smaller than the P-factor and R-factor ratios of 1.00 in the fourth iteration of the MS-CUA method.

Therefore, the SUFI-2 method can provide slightly better-calibrated simulation results, and the MS-CUA method can give more balanced uncertainty analysis results. To be noticed, if only three iterations have been conducted, the MS-CUA method can still provide reasonably good uncertainty analysis results $(\mathrm{P} / \mathrm{R}=0.93)$; at the same time, the SUFI-2 method only provided relatively poor uncertainty analysis results $(\mathrm{P} / \mathrm{R}=0.61)$. The MSCUA method can more efficiently achieve a desired uncertainty analysis results with a reasonably well-calibrated simulation result. When less computational resources were provided, the MS-CUA method can make quick and reliable responses than the SUFI-2 method.

\section{- GLUE}

GLUE was also used for calibration and uncertainty analysis for comparison purposes. A total of 10,000 simulation runs was conducted. The initial parameter ranges for the MSCUA method were used as the parameter ranges for each parameter in GLUE. The NSE was used as the likelihood function to keep consistency with the other two methods, and the threshold value was set to 0.60 because a large number of simulation runs was conducted. Table 6 shows the calibration and uncertainty analysis results by using the GLUE method. As shown in Table 6, there were only 28 behavioral parameter sets among the total of 10,000 parameter sets, representing the low efficiency of searching the optimal results using GLUE. The $\mathrm{R}^{2}$ and NSE value were 0.79 and 0.70 , respectively, which were smaller than the results from the fourth iteration of the MS-CUA method $\left(\mathrm{R}^{2}=0.79\right.$ and $\mathrm{NSE}=0.74)$. The $\mathrm{P} / \mathrm{R}$ ratio was 0.76 lower than the value of the MS-CUA method $(\mathrm{P} / \mathrm{R}=$ 1.00 ), indicating that the proposed method can provide a better-calibrated simulation with better uncertainty analysis results. Meanwhile, only 4,000 simulation runs were conducted to achieve better calibration and uncertainty analysis for the MS-CUA method compared with the 10,000 simulation runs in GLUE, demonstrating the advantages of the proposed method. 
According to the results from three methods, it was clearly shown that the MS-CUA method can reach similar good calibration results with SUFI-2 (which was better than GLUE) and provided the best uncertainty analysis results among the three methods. The MS-CUA method also showed the ability to efficiently search the HPD region and reduce the prediction uncertainty of surface runoff and parameter uncertainty while demonstrating its advantages over other methods.

Table 5 Parameter range values of each iteration using SUFI-2

Table 6 The statistic summary of the simulation results of each iteration using SUFI-2

\section{Conclusions}

In this study, a developed calibration and uncertainty analysis method (MS-CUA) was proposed for hydrological modeling studies. The MS-CUA method with the proposed framework aimed to calibrate hydrological models and provide the balanced and reliable uncertainty analysis results in a high-efficient way. The feasibility and flexibility of MSCUA method were evaluated through two case studies based on the simulations from the SWAT model. A comprehensive analysis was achieved according to the hypothetical case results with demo data from SWAT-CUP and the real case study at upstream of the Wenjing River watershed.

In the hypothetical case study, the results indicated that with 2,000 simulation runs the MSCUA method can achieve better values of $R^{2}, N S E$ and $P / R$ ratio than those in 10,000 simulation runs by GLUE. In the real-world case study, the 4,000 simulation runs using the MS-CUA method were compared with 4,000 simulation runs using SUFI-2 and 10,000 simulation runs using GLUE. The MS-CUA method can provide better uncertainty analysis results than the SUFI-2 method with similarly well-calibrated simulations and achieve more balanced uncertainty analysis results with fewer simulation runs. The MS-CUA method obtained better calibration results and much better uncertainty analysis results with fewer simulation runs with the comparison of GLUE. The results indicated that the MSCUA method can search optimal simulations and provide balanced uncertainty analysis results with high efficiency.

Through the case studies, the results showed that the MS-CUA method was able to locate the HPD regions of each parameter fast for the improvement of the computational efficiency and reduction of the parameter uncertainty without sacrificing the simulation performance for surface runoff prediction. Through the addition criterion (R2) application, behavioral parameter sets were further screened for the refined behavioral parameter sets in each iteration. The reduced number of behavioral parameter sets can decrease the influence of equifinality phenomenon showing the unique advantage of the MS-CUA method. The MS-CUA method could be applied to the high-dimensional parameter estimation problems and complex simulation models due to the high computational efficiency. The parameter ranges in the MS-CUA method are always centered on the best simulation results and narrowed down from the original ranges with the use of refined behavioral parameter sets. Thus, the parameter uncertainty has been reduced after each iteration. The reduced parameter uncertainty was quite important when conducting the 
uncertainty analysis for propagation effects. The less uncertainty from the source would lead to much smaller total uncertainty after propagation for future studies. The proposed MS-CUA approach also has the potential to deal with the problems related to calibration and uncertainty analysis of other simulation models.

\section{Methods}

\section{- The MS-CUA method}

Using advanced sampling methods for prior distributions and new criteria for screening behavioral parameter sets for posterior distributions, narrower and more reliable parameter uncertainty ranges could be provided for improving the simulation performance. The framework (Figure 4) and detailed procedures of the MS-CUA method are shown as follows:

Figure 4. The framework of the MS-CUA method for calibration and uncertainty

Step 1: The selection of likelihood functions. As a popular likelihood function, NashSutcliffe Efficiency (NSE) (Eq. 1) can be selected ${ }^{34,35}$. The initial sample sizes should be defined at this step. Due to the application of multiple iterations and high-efficiency sampling methods, relatively small sample sizes for each iteration are used.

$$
N S E=1-\frac{\sum_{i=1}^{n}\left(Q_{s, i}-Q_{o, i}\right)^{2}}{\sum_{i=1}^{n}\left(Q_{o, i}-\overline{Q_{o}}\right)^{2}}
$$

where, $Q_{o, i}$ and $Q_{s, i}$ are the observed and simulated values on day $i$, respectively; $\bar{Q}_{o}$ and $\bar{Q}_{i}$ are the average values of the observed and simulated surface runoff $\left(\mathrm{m}^{3} / \mathrm{s}\right)$, respectively; and $n$ is the total number of values within the period of analysis.

Step 2: The definition of acceptable percentage levels. Decide the percentage of observations within uncertainty ranges and the percentage of refined behavioral parameter sets among total behavioral parameter sets. If the selected number of behavioral parameter sets is too small, the parameter ranges for the next iteration may not be accurate. Therefore, an appropriate percentage of behavioral parameters is necessary to ensure a reasonably good estimation for the next iteration.

Step 3: The determination of parameter ranges. Parameter ranges can be assigned according to the physical meaning and current understanding of parameters. And then, advanced sampling schemes methods of Latin hypercube sampling (LHS) and the Shuffled complex evolution (SCE-UA) method are applied. Due to missing information, the prior distributions of parameters are generally assumed as uniform distributions. It is a typical assumption in hydrological modeling, because the prior distribution form of parameters was normally difficult to determine ${ }^{36}$.

Step 4: The definition of threshold values of the likelihood function. The threshold values of the likelihood function are defined for screening the behavioral and non-behavioral parameter sets. A higher value of the objective function threshold typically achieves 
smaller numbers of behavioral parameter sets. For different iterations, multiple choices of likelihood function values can be applied for a better estimation of parameter ranges. For example, relatively low threshold values can be selected for the first iteration due to the lack of information.

Step 5: The computation of hydrological simulation and likelihood function values. Hydrological simulations can be conducted using predefined parameter sets. And then, the likelihood function values of simulations from each parameter sets are calculated. The simulation results from parameter sets are further ranked based on their likelihood values. The parameter sets with likelihood function values lower than the threshold value are removed. The remaining parameter sets are defined as the behavioral parameter sets.

Step 6: The application of additional criteria. Additional criteria are to screen out the "bad" behavioral simulations, even though their NSE values are greater than the predefined threshold value. After removing the behavioral parameter sets with the additional criteria, the remaining parameter sets are called "refined behavioral parameter sets". For example, the coefficient of determination $\left(R^{2}\right)$ can be used as an additional criterion for screening refined behavioral parameter sets (Eq. 2). Moreover, with the involvement of new constraints with new information (e.g., new observations can lead to changes of some parameter ranges), some behavioral parameter sets will be removed. Eventually, the number of refined behavioral parameter sets will be reduced and determined, and prediction uncertainty will be reduced and quantified correspondingly. By using the additional criteria, the influence of equifinality phenomenon can be controlled and reduced through the refining process using the above steps. In certain cases, some attempts of additional criteria (Eqs. 2, 3, and 4) can be considered when data are available as follows:

$$
\begin{aligned}
& R^{2}=\frac{\left[\sum_{i=1}^{n}\left(Q_{o, i}-\overline{Q_{o}}\right)\left(Q_{s, i}-\overline{Q_{s}}\right)\right]^{2}}{\sum_{i=1}^{n}\left(Q_{o, i}-\bar{Q}_{o}\right)^{2} \sum_{i=1}^{n}\left(Q_{s, i}-\bar{Q}_{s}\right)^{2}} \\
& L_{1}=\frac{1}{n_{p}} \sum_{j=1}^{n_{p}}\left|\frac{Q_{o, j}-Q_{s, j}}{Q_{o, j}}\right|
\end{aligned}
$$

where, $n_{p}$ is the number of peak flows during the study period. It can be assumed that the average difference between observed peak flow and simulated peak flow cannot be more than $30 \%$ for a behavioral simulation. Therefore, for parameter sets have $L_{1}$ the greater than $30 \%$, these parameter sets will be removed from total behavioral parameter sets. The remaining parameter sets are considered as refined behavioral parameter sets.

$$
L_{2}=\frac{1}{n_{p}} \sum_{j=1}^{n_{p}}\left|t_{o, j}-t_{s, j}\right|
$$

where, $t_{o, j}$ is the observed time for accessing the peak flow (hour), $t_{s, j}$ is the simulated time for accessing peak flow (hour). For example, if difference between the time to peak flow in observation and in simulation results is assumed than less than 3 hours. In that case, any parameter sets making $L_{2}$ greater than 3 hours will be removed to obtain the refined 
behavioral parameter sets.

Step 7: The analysis of model prediction uncertainty. Model prediction uncertainty analysis can be conducted in this step. The lower and upper bounds of model prediction results are determined for surface runoff. By sorting the likelihood values of simulations from each parameter sets, the time series surface runoff prediction uncertainty under the given confidence level could be estimated. For most studies, $2.5 \%$ and $97.5 \%$ of the cumulative distribution of surface runoff are set as the lower and upper bounds as prediction uncertainty, respectively. Therefore, 95 percent prediction uncertainty (95PPU) of the surface runoff simulation is obtained through the analysis. For the next iteration, the parameter ranges are updated accordingly using the parameter ranges for the simulations achieving the 95PPU of surface runoff. Since the number of refined behavioral parameter sets is continuously reduced after each iteration, the corresponding parameter ranges will be further reduced. After the update of parameter ranges, go back to Step 5 for the new iteration till the desired numbers of iteration are completed.

Step 8: The determination of the desired percentage of observation. When 95PPU values of surface runoff are obtained, the desired percentage of observation within the 95PPU can be determined. If the percentage is too low and cannot meet the pre-set value, go back to Step 4: change the threshold value of the likelihood function to a lower one or adjust the critical value of other criteria to increase the behavioral parameter sets for getting a reasonable estimation of parameter ranges. If the percentage of observation becomes reasonable, the next step can proceed.

Step 9: The validation of the proposed method. Validation of the proposed method is necessary. After obtaining the parameter ranges from the last iteration of simulations, the newly updated parameter ranges are applied for one more iteration to check if the reasonable outcomes are achieved. If simulation results are acceptable, go to the last step; if not, go back to Step 4.

Step 10: The results of calibration and uncertainty analysis. This proposed method uses multiple iterations with advanced sampling methods and screens out the "bad" behavioral parameter sets using additional criteria to efficiently and accurately search the optimal results with consideration of uncertainty. The reduced parameter ranges also lead to smaller parameter uncertainty reflected by 95PPU of the surface runoff. The highly efficient calibration and uncertainty analysis can be achieved.

The MS-CUA method is different from the GLUE method, which accepts all the parameter sets as behavioral parameter sets if the likelihood values are greater than the predefined threshold value. To our knowledge, limited studies have focused on refining the posterior distribution of parameters to make the simulation more accurate. The GLUE method generally applies only one likelihood function, which should increase monotonically with the similarity in behavior increase (e.g. Nash-Sutcliffe coefficient) ${ }^{10}$. The objective of the GLUE method is to identify a set of behavioral models within the range of possible parameter combinations. The term "behavioral" indicates acceptable models based on available data and knowledge. Therefore, a threshold value needs to be predefined before 
screening the behavioral and non-behavioral parameter sets. However, some parameter sets can achieve a high value for the likelihood function but are still not reasonable in practice. Because the NSE only evaluates the goodness-of-fit of the overall runoff simulation, some impractical and inaccurate simulation results are involved in behavioral results. For example, it could have an NSE value over 0.80 for the overall simulation for runoff, but the simulated peak flow is quite different from the observed peak flow; or the time for the peak flow in simulation is much different from the time for the corresponding peak flow in observation. On the other hand, similar to the sequential uncertainty fitting version 2 (SUFI-2) method, the MS-CUA implement multiple iterations for the performance improvements of simulation and uncertainty analysis. However, different updating algorithms of parameter ranges are applied in each iteration.

\section{- Two sampling schemes}

In the proposed methods, instead of using the Monte Carlo random sampling method for prior distribution sampling of model parameters, advanced sampling methods are applied for parameter prior distributions to improve sampling efficiency. The sampling methods can be Latin hypercube sampling (LHS) and shuffled complex evolution (SCE-UA).

\section{(a) Latin hypercube sampling (LHS)}

The LHS is a probabilistic procedure with the combinations of multiple desired features of random sampling and stratified sampling for more stable analysis outcomes rather than random sampling ${ }^{37}$. Many studies have proved that the LHS is an efficient way for assessing output uncertainty of models (e.g., most distributed hydrological model) with multiple parameters ${ }^{38-40}$. This technique applies a stratified sampling scheme, which allows an efficient description of the output. The standard LHS method contains three major steps as follow ${ }^{41}$ :

Step 1: Equiprobable subdivision: The probability distribution of parameters in each model is subdivided into $T$ ranges/intervals with a probability of occurrence equal to $1 / T$, respectively.

Step 2: Stratified sampling: A single value is sampled within each range/interval according to the probability distribution (normally, the uniform distribution is assumed for parameters).

Step 3: Random pairing: $T$ data sets of $p$ parameters ( $p$ is the number of parameters to be sampled) are created.

The LHS method represents a better performance with a more uniform coverage of parameter space than other methods in estimating the statistics of a population of function with less model simulations ${ }^{42}$. Moreover, the LHS is easy to be implemented. Therefore, using the LHS method, the high-efficient sampling for uncertainty analysis can be relatively easy to achieve.

\section{(b) Shuffled complex evolution (SCE-UA):}

The SCE-UA algorithm, developed by Duan, et al. ${ }^{43}$, is a robust global search algorithm to sample parameter sets for prior distribution. Originally, this method is used to minimize a single objective function on the basis of four concepts: 1) the combination of deterministic and probabilistic methods; 2) the ability for a systematic evolution for the parameter space to the global optimum; 3) multiple competitive evolutions; 4) complex 
shuffling in each community. Because of these features, the SCE-UA method is effective, robust, and flexible. The major steps of the SCE-UA method are described below ${ }^{44,45}$ :

Step 1: Generate samples: Sample $s$ points randomly in the reasonable parameter range and compute the likelihood value at each point. Typically, the uniform probability distribution will be applied for the sample generation if lacking prior information.

Step 2: Rank samples: Sort the $s$ points in ascending order of likelihood values.

Step 3: Partition into complexes: Partition $s$ points into $p$ groups (called complexes), and each complex contains $m$ points. The complexes are partitioned so that the first complex contains every $p(k-1)+1$ ranked point, the second complex contains every $p(k-1)+2$ ranked point and so on, where $k=1,2, \ldots, m$.

Step 4: Evolve each complex: Evolve each complex according to the competitive complex evolution (CCE) algorithm.

Step 5: Shuffle complexes: combine the points in the evolved complexes into a single sample population and sort the sample population by ascending sequence according to likelihood values.

Step 6: Check convergence: If any of the pre-specified convergence criteria are satisfied, then stop; otherwise, then continue.

Step 7: Check the reduction of the number of complexes: If the minimum number of complexes required in a population $\left(p_{\min }\right)$ is less than $p$, remove the lowest-ranked complex; set $p=p-1$ and $s=p m$; return to Step 4; If $p_{\min }=p$, go back to Step 4 .

By using the SCE-UA method, considerable improvements can be made due to an adaptive sampling method that uses information from past draws to update the search direction. In such a way, SCE-UA can generate a more robust and efficient estimation for parameters and prediction uncertainty in comparison to a traditional random Monte Carlo sampling method.

\section{- Hydrological modeling}

The hydrological modeling was conducted using the soil and water assessment tool (SWAT), and the simulation results are used for analysis. SWAT is a continuous-time, spatially distributed hydrological model. It was first developed by the United States Department of Agriculture-Agricultural Research Service (USDA-ARS) ${ }^{46}$. SWAT can help water resource managers predict the impacts of land management practice on water, sediment, and agricultural chemical yields ${ }^{47,48}$. The SWAT model uses watershed information (e.g. weather, soil, topography, vegetation and land management practices) to simulate watershed hydrological processes such as surface and subsurface runoff, water quality, erosion and sedimentation ${ }^{49}$. One of the advantages of the SWAT model is the capability of simulations in ungaged river basins. By considering data availability, SWAT has been selected for this case study.

\section{References}

1 Karlsson, I. B. et al. Combined effects of climate models, hydrological model structures and land use scenarios on hydrological impacts of climate change. Journal of Hydrology 535, 301-317 (2016).

2 Wang, J. et al. Application of BP neural network algorithm in traditional 
hydrological model for flood forecasting. Water $\mathbf{9}, 48$ (2017).

3 Lindenschmidt, K. \& Rokaya, P. A Stochastic Hydraulic Modelling Approach to Determining the Probable Maximum Staging of Ice-Jam Floods. Journal of Environmental Informatics 34 (2019).

4 Stryker, J., Wemple, B. \& Bomblies, A. Modeling sediment mobilization using a distributed hydrological model coupled with a bank stability model. Water Resources Research 53, 2051-2073 (2017).

5 Garnier, J. et al. Nutrient inputs and hydrology together determine biogeochemical status of the Loire River (France): Current situation and possible future scenarios. Science of the Total Environment 637, 609-624 (2018).

$6 \mathrm{Wu}, \mathrm{H}$., Chen, B., Snelgrove, K. \& Lye, L. Quantification of uncertainty propagation effects during statistical downscaling of precipitation and temperature to hydrological modeling. J. Environ. Inform 34, 139-148 (2019).

7 Qi, W., Zhang, C., Fu, G., Sweetapple, C. \& Liu, Y. Impact of robustness of hydrological model parameters on flood prediction uncertainty. Journal of Flood Risk Management 12, e12488 (2019).

8 Huo, J. \& Liu, L. Application research of multi-objective Artificial Bee Colony optimization algorithm for parameters calibration of hydrological model. Neural Computing and Applications 31, 4715-4732 (2019).

9 Chen, Y., Li, J. \& Xu, H. Improving flood forecasting capability of physically based distributed hydrological models by parameter optimization. Hydrology and Earth System Sciences 20, 375 (2016).

$10 \mathrm{Wu}, \mathrm{H}$. \& Chen, B. Evaluating uncertainty estimates in distributed hydrological modeling for the Wenjing River watershed in China by GLUE, SUFI-2, and ParaSol methods. Ecological engineering 76, 110-121 (2015).

11 Bai, P., Liu, X. \& Liu, C. Improving hydrological simulations by incorporating GRACE data for model calibration. Journal of Hydrology 557, 291-304 (2018).

12 Van Stralen, P. \& Pimentel, A. in ARCS 2012. 1-7 (IEEE).

13 Bajracharya, A., Awoye, H., Stadnyk, T. \& Asadzadeh, M. Time Variant Sensitivity Analysis of Hydrological Model Parameters in a Cold Region using Flow Signatures. Water 12, 961 (2020).

14 Pianosi, F. \& Wagener, T. Understanding the time - varying importance of different uncertainty sources in hydrological modelling using global sensitivity analysis. Hydrological Processes 30, 3991-4003 (2016).

15 Liu, Y., Li, Y., Huang, G., Zhang, J. \& Fan, Y. A Bayesian-based multilevel factorial analysis method for analyzing parameter uncertainty of hydrological model. Journal of Hydrology 553, 750-762 (2017).

16 Kan, G. et al. Heterogeneous parallel computing accelerated generalized likelihood uncertainty estimation (GLUE) method for fast hydrological model uncertainty analysis purpose. Engineering with Computers 36, 75-96 (2020).

17 Her, Y. et al. Uncertainty in hydrological analysis of climate change: multiparameter vs. multi-GCM ensemble predictions. Scientific reports 9, 1-22 (2019).

18 Wu, Q., Liu, S., Cai, Y., Li, X. \& Jiang, Y. Improvement of hydrological model calibration by selecting multiple parameter ranges. Hydrology and Earth System Sciences 21, 393 (2017).

19 Westerberg, I. K., Sikorska-Senoner, A. E., Viviroli, D., Vis, M. \& Seibert, J. 
Hydrological model calibration with uncertain discharge data. Hydrological Sciences Journal, 1-16 (2020).

20 Halpern, J. Y. Reasoning about uncertainty. (MIT press, 2017).

21 Ghanem, R., Higdon, D. \& Owhadi, H. Handbook of uncertainty quantification. Vol. 6 (Springer, 2017).

22 Chen, L., Han, Z., Wang, G. \& Shen, Z. Uncertainty analysis for an effluent trading system in a typical nonpoint-sources-polluted watershed. Scientific reports $\mathbf{6}, 1-12$ (2016).

23 Abbaspour, K. C. SWAT Calibration and Uncertainty Programs - A User Manual. Eawag: Swiss Federal Institute of Aquatic Science and Technology. (2015).

24 Mushtaq, F., Bland, A. R. \& Schaefer, A. Uncertainty and cognitive control. Frontiers in psychology 2, 249 (2011).

25 Li, Z., Shao, Q., Xu, Z. \& Cai, X. Analysis of parameter uncertainty in semidistributed hydrological models using bootstrap method: A case study of SWAT model applied to Yingluoxia watershed in northwest China. Journal of Hydrology 385, 76-83 (2010).

26 Abbaspour, K. C., Johnson, C. A. \& Van Genuchten, M. T. Estimating uncertain flow and transport parameters using a sequential uncertainty fitting procedure. Vadose Zone Journal 3, 1340-1352 (2004).

27 Narsimlu, B., Gosain, A. K., Chahar, B. R., Singh, S. K. \& Srivastava, P. K. SWAT model calibration and uncertainty analysis for streamflow prediction in the Kunwari River Basin, India, using sequential uncertainty fitting. Environmental Processes 2, 79-95 (2015).

28 Abbas, T. et al. Uncertainty analysis of runoff and sedimentation in a forested watershed using sequential uncertainty fitting method. Sciences in Cold and Arid Regions 8, 297-310 (2016).

29 Joh, H.-K., Park, J.-Y., Jang, C.-H. \& Kim, S.-J. Comparing prediction uncertainty analysis techniques of SWAT simulated streamflow applied to Chungju dam watershed. Journal of Korea Water Resources Association 45, 861-874 (2012).

30 Dodwell, T. J., Ketelsen, C., Scheichl, R. \& Teckentrup, A. L. A hierarchical multilevel Markov chain Monte Carlo algorithm with applications to uncertainty quantification in subsurface flow. SIAM/ASA Journal on Uncertainty Quantification 3, 1075-1108 (2015).

31 He, J., Jones, J. W., Graham, W. D. \& Dukes, M. D. Influence of likelihood function choice for estimating crop model parameters using the generalized likelihood uncertainty estimation method. Agricultural Systems 103, 256-264 (2010).

32 Mishra, A. et al. Generalized likelihood uncertainty estimation and Markov chain Monte Carlo simulation to prioritize TMDL pollutant allocations. Journal of Hydrologic Engineering 23, 05018025 (2018).

33 Winchell, M., Srinivasan, R., Luzio, M. D. \& Arnold., J. (Grassland, soil and water research service, Texas, the United States, 2009).

$34 \mathrm{Wu}, \mathrm{H}$., Lye, L. M. \& Chen, B. A design of experiment aided sensitivity analysis and parameterization for hydrological modeling. Canadian Journal of Civil Engineering 39, 460-472, doi:10.1139/12012-017 (2012).

35 Nash, J. E. \& Sutcliffe, J. V. River flow forecasting through conceptual models part I-A discussion of principles. Journal of hydrology 10, 282-290 (1970). 
36 Blasone, R.-S. et al. Generalized likelihood uncertainty estimation (GLUE) using adaptive Markov Chain Monte Carlo sampling. Advances in Water Resources 31, 630-648, doi:10.1016/j.advwatres.2007.12.003 (2008).

37 Helton, J. C. \& Davis, F. J. Latin hypercube sampling and the propagation of uncertainty in analyses of complex systems. Reliability Engineering \& System Safety 81, 23-69 (2003).

38 Christiaens, K. \& Feyen, J. Constraining soil hydraulic parameter and output uncertainty of the distributed hydrological MIKE SHE model using the GLUE framework. Hydrological Processes 16, 373-391 (2002).

39 Dunn, M. C., Shotorban, B. \& Frendi, A. Uncertainty quantification of turbulence model coefficients via latin hypercube sampling method. Journal of fluids engineering 133 (2011).

40 Hansen, C. W., Helton, J. C. \& Sallaberry, C. J. Use of replicated Latin hypercube sampling to estimate sampling variance in uncertainty and sensitivity analysis results for the geologic disposal of radioactive waste. Reliability Engineering \& System Safety 107, 139-148 (2012).

41 Janssen, P., Heuberger, P. \& Sanders, R. UNCSAM 1.1: A Software Package for Sensitivity and Uncertainty Analysis. Manual. RIVM Rapport 959101004 (1992).

42 Blasone, R.-S. (Kgs. Lyngby, 2007).

43 Duan, Q. y., Sorooshian, S. \& Gupta, V. Effective and efficient global optimization for conceptual rainfall-runoff models. Water Resour. Res 28, 1015-1031 (1992).

44 Duan, Q. y., Sorooshian, S. \& Gupta, V. K. Optimal use of the SCE-UA global optimization method for calibrating watershed models. Journal of hydrology 158, 265-284 (1994).

45 Kan, G. et al. A heterogeneous computing accelerated SCE-UA global optimization method using OpenMP, OpenCL, CUDA, and OpenACC. Water Science and Technology 76, 1640-1651 (2017).

46 Arnold, J. G., Srinivasan, R., Muttiah, R. S. \& Williams, J. R. Large area hydrologic modeling and assessment Part I: Model development. Journal of the American Water Resources Association 34, 17 (1998).

47 Neitsch, S. L., Arnold, J. G., Kiniry, J. R. \& Williams, J. R. Soil and Water Assessment Tool Theoretical Documentation, Version 2009. Grassland, soil and water research service, Texas, the United States. (2011).

48 Francesconi, W., Srinivasan, R., Pérez-Miñana, E., Willcock, S. P. \& Quintero, M. Using the Soil and Water Assessment Tool (SWAT) to model ecosystem services: A systematic review. Journal of Hydrology 535, 625-636 (2016).

49 Muleta, M. K. \& Nicklow, J. W. Sensitivity and uncertainty analysis coupled with automatic calibration for a distributed watershed model. Journal of Hydrology $\mathbf{3 0 6}$, 127-145 (2005).

\section{Acknowledgements}

Special thanks go to the Natural Sciences and Engineering Research Council of Canada (NSERC), the Canada Foundation for Innovation (CFI), the Canada-China Scholars Exchange Program (CCSEP), the Department of Foreign Affairs, Trade and Development Canada (DFATD), the Department of Tourism, Culture, Industry and Innovation (TCII), the United Nations Development Programme (UNDP), the local authorities in China, and the NSERC CREATE Network on Persistent, Emerging and Organic Pollution in the 
Environment (PEOPLE Network).for the funding and technical support.

\section{Author contribution statement}

H.W. and X.Y. organized and wrote the manuscript. H.W. and B.C. developed the methodology. H.W. collected and analyzed the data. X.Y. assisted in analyzed the data. All authors contributed to discussing the results. All authors reviewed the manuscript.

\section{Additional information}

Competing Interests: The authors declare that they have no competing interests.

\section{- The list of tables}

Table 1. Definitions of selected input parameters in SWAT $^{33}$

\begin{tabular}{ll}
\hline CN2 & Initial SCS runoff curve number for moisture condition II. \\
ALPHA_BF & Baseflow alpha factor (days). \\
GW_DELAY: & The delay time, $\delta_{\text {gw }}$, cannot be directly measured. \\
GWQMN: & Threshold depth of water in the shallow aquifer required for return \\
& flow to occur (mm H20). \\
ESCO & Soil evaporation compensation factor. \\
CH_N2 & Manning's "n" value for the main channel \\
CH_K2: & Effective hydraulic conductivity in main channel alluvium $(\mathrm{mm} / \mathrm{hr})$. \\
ALPHA_BNK & Baseflow alpha factor for bank storage $($ days). \\
SOL_AWC: & Available water capacity of the soil layer $(\mathrm{mm} \mathrm{H} 2 \mathrm{O} / \mathrm{mm}$ soil). \\
SOL_K & Saturated hydraulic conductivity for the first soil layer \\
SOL_BD & Moist bulk density for the first soil layer \\
SFTMP: & Snowfall temperature $\left({ }^{\circ} \mathrm{C}\right)$. \\
GW_REVAP & Groundwater "revap" coefficient. \\
RCHRG_DP: & Deep aquifer percolation fraction. \\
\hline
\end{tabular}

Table 2 The comparison results between the simplified MS-CUA method and GLUE

\begin{tabular}{|c|c|c|c|c|c|c|c|}
\hline Outlet & Variable & $\begin{array}{l}\text { Behavioral } \\
\text { simulation }\end{array}$ & $\begin{array}{c}P- \\
\text { factor }\end{array}$ & $\begin{array}{c}\text { R- } \\
\text { factor }\end{array}$ & $\mathbf{R}^{2}$ & NSE & $\mathbf{P} / \mathbf{R}$ \\
\hline \multirow{3}{*}{1} & $\begin{array}{l}\text { 1st iteration of MS- } \\
\text { CUA }\end{array}$ & $484(1000)$ & 0.38 & 0.31 & 0.89 & 0.88 & 1.23 \\
\hline & $\begin{array}{l}\text { 2nd iteration of MS- } \\
\text { CUA }\end{array}$ & $976(1000)$ & 0.38 & 0.3 & 0.91 & 0.91 & 1.23 \\
\hline & GLUE & $7660(10000)$ & 0.38 & 0.53 & 0.91 & 0.90 & 0.72 \\
\hline & $\begin{array}{c}\text { 1st iteration of MS- } \\
\text { CUA }\end{array}$ & $484(1000)$ & 0.58 & 0.27 & 0.97 & 0.96 & 2.15 \\
\hline & $\begin{array}{l}\text { 2nd iteration of MS- } \\
\text { CUA }\end{array}$ & $976(1000)$ & 0.58 & 0.27 & 0.98 & 0.97 & 2.15 \\
\hline & GLUE & $7660(10000)$ & 0.63 & 0.47 & 0.97 & 0.96 & 1.34 \\
\hline
\end{tabular}

*P-factor: the percentage of observations covered by the 95PPU.

$* \mathrm{R}$-factor: the measure of the relative width of $95 \%$ probability band.

Table 3 The statistic summary of the simulation results 


\begin{tabular}{|c|c|c|c|c|c|c|c|c|c|}
\hline Scenario & Iteration & $\begin{array}{l}\text { No. of } \\
\text { criteria }\end{array}$ & $\begin{array}{l}\text { Threshold } \\
\text { value }\end{array}$ & $\begin{array}{c}\text { Behavioral } \\
\text { parameter } \\
\text { sets }\end{array}$ & $\begin{array}{c}\text { P- } \\
\text { factor }\end{array}$ & $\begin{array}{c}\text { R- } \\
\text { factor }\end{array}$ & $\mathbf{R}^{2}$ & NSE & $\mathbf{P} / \mathbf{R}$ \\
\hline \multirow{4}{*}{ 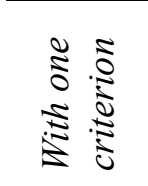 } & First & - & 0.20 & 86 & 0.50 & 0.90 & 0.78 & 0.68 & 0.56 \\
\hline & Second & - & 0.40 & 61 & 0.50 & 0.78 & 0.78 & 0.70 & 0.64 \\
\hline & Third & - & 0.60 & 49 & 0.47 & 0.54 & 0.77 & 0.70 & 0.87 \\
\hline & Fourth & - & 0.60 & 410 & 0.42 & 0.49 & 0.77 & 0.71 & 0.86 \\
\hline \multirow{8}{*}{ 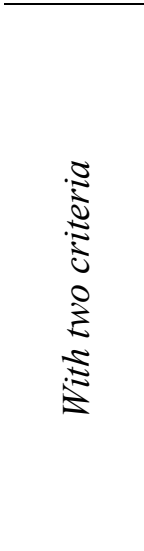 } & \multirow[b]{2}{*}{ First } & One & $\mathrm{NSE}=0.20$ & 86 & 0.50 & 0.90 & 0.78 & 0.68 & 0.56 \\
\hline & & Two & $\begin{array}{c}\mathrm{NSE}=0.20 \\
\mathrm{R}^{2}=0.60\end{array}$ & 81 & 0.50 & 0.90 & 0.78 & 0.68 & 0.56 \\
\hline & \multirow[b]{2}{*}{ Second } & One & $\mathrm{NSE}=0.40$ & 62 & 0.47 & 0.71 & 0.78 & 0.70 & 0.66 \\
\hline & & Two & $\begin{array}{c}\mathrm{NSE}=0.40 \\
\mathrm{R}^{2}=0.70\end{array}$ & 34 & 0.47 & 0.66 & 0.78 & 0.70 & 0.71 \\
\hline & \multirow[b]{2}{*}{ Third } & One & $\mathrm{NSE}=0.60$ & 74 & 0.44 & 0.52 & 0.78 & 0.73 & 0.85 \\
\hline & & Two & $\begin{array}{c}\mathrm{NSE}=0.60 \\
\mathrm{R}^{2}=0.75\end{array}$ & 41 & 0.42 & 0.45 & 0.78 & 0.73 & 0.93 \\
\hline & \multirow[b]{2}{*}{ Fourth } & One & $\mathrm{NSE}=0.60$ & 605 & 0.39 & 0.39 & 0.79 & 0.74 & 1.00 \\
\hline & & Two & $\begin{array}{c}\mathrm{NSE}=0.60 \\
\mathrm{R}^{2}=0.75\end{array}$ & 527 & 0.39 & 0.39 & 0.79 & 0.74 & 1.00 \\
\hline
\end{tabular}

Table 4 Parameter range values of each iteration

\begin{tabular}{|c|c|c|c|c|c|c|c|c|c|}
\hline \multirow{2}{*}{ Parameter } & \multirow{2}{*}{ Scenario* } & \multicolumn{2}{|c|}{$\begin{array}{c}\text { First } \\
\text { iteration }\end{array}$} & \multicolumn{2}{|c|}{$\begin{array}{l}\text { Second } \\
\text { iteration }\end{array}$} & \multicolumn{2}{|c|}{$\begin{array}{c}\text { Third } \\
\text { iteration }\end{array}$} & \multicolumn{2}{|c|}{$\begin{array}{l}\text { Fourth } \\
\text { iteration }\end{array}$} \\
\hline & & $\mathbf{L B}^{* *}$ & UB & LB & UB & LB & UB & LB & UB \\
\hline \multirow{2}{*}{$\mathrm{CN}_{2}$} & 1 & -0.25 & 0.30 & -0.22 & 0.29 & -0.19 & 0.28 & -0.19 & 0.13 \\
\hline & 2 & -0.25 & 0.30 & -0.22 & 0.29 & -0.18 & 0.25 & -0.18 & 0.07 \\
\hline \multirow{2}{*}{ ALPHA_BF } & 1 & 0.40 & 1.00 & 0.43 & 0.99 & 0.44 & 0.97 & 0.48 & 0.92 \\
\hline & 2 & 0.40 & 1.00 & 0.43 & 0.99 & 0.47 & 0.96 & 0.52 & 0.95 \\
\hline \multirow{2}{*}{ GW_DELAY } & 1 & 10.00 & 300.00 & 13.84 & 280.90 & 25.73 & 248.05 & 33.21 & 155.65 \\
\hline & 2 & 10.00 & 300.00 & 13.63 & 281.30 & 22.83 & 169.09 & 38.56 & 102.33 \\
\hline \multirow{2}{*}{ GWQMN } & 1 & 0.00 & 2000.00 & 13.75 & 1358.25 & 21.82 & 586.51 & 31.45 & 429.00 \\
\hline & 2 & 0.00 & 2000.00 & 13.00 & 1375.00 & 54.03 & 505.09 & 81.32 & 446.23 \\
\hline \multirow{2}{*}{ ESCO } & 1 & 0.80 & 1.00 & 0.81 & 0.99 & 0.83 & 0.99 & 0.84 & 0.99 \\
\hline & 2 & 0.80 & 1.00 & 0.81 & 0.99 & 0.82 & 0.99 & 0.82 & 0.98 \\
\hline \multirow{2}{*}{ CH_K } & 1 & 5.00 & 130.00 & 10.81 & 128.17 & 16.45 & 121.83 & 19.10 & 118.34 \\
\hline & 2 & 5.00 & 130.00 & 10.31 & 128.31 & 14.89 & 120.62 & 16.84 & 117.72 \\
\hline \multirow{2}{*}{ ALPHA_BNK } & 1 & 0.00 & 1.00 & 0.04 & 0.98 & 0.062 & 0.95 & 0.14 & 0.92 \\
\hline & 2 & 0.00 & 1.00 & 0.04 & 0.98 & 0.088 & 0.88 & 0.12 & 0.83 \\
\hline \multirow{2}{*}{ SOL_AWC } & 1 & -0.20 & 0.40 & -0.18 & 0.39 & -0.13 & 0.38 & -0.12 & 0.36 \\
\hline & 2 & -0.20 & 0.40 & -0.17 & 0.39 & -0.15 & 0.34 & -0.12 & 0.32 \\
\hline
\end{tabular}




\begin{tabular}{cccccccccc}
\hline \multirow{2}{*}{ SFTMP } & 1 & -5.00 & 5.00 & -4.46 & 4.70 & -4.07 & 4.38 & -4.01 & 3.99 \\
& 2 & -5.00 & 5.00 & -4.25 & 4.71 & -4.02 & 4.59 & -3.89 & 4.01 \\
GW_REVAP & 1 & 0.02 & 0.50 & 0.02 & 0.44 & 0.026 & 0.24 & 0.03 & 0.15 \\
& 2 & 0.02 & 0.50 & 0.02 & 0.44 & 0.037 & 0.26 & 0.04 & 0.12 \\
RCHRG_DP & 1 & 0.00 & 1.00 & 0.0080 & 0.64 & 0.014 & 0.37 & 0.0145 & 0.19 \\
& 2 & 0.00 & 1.00 & 0.0075 & 0.64 & 0.019 & 0.34 & 0.0348 & 0.19 \\
\hline
\end{tabular}

* Scenarios: 1, simulations with one criterion; 2, simulations with two criteria.

** LB: Lower bound; UB: Upper bound.

Table 5 Parameter range values of each iteration using SUFI-2

\begin{tabular}{ccccccccc}
\hline \multirow{2}{*}{ Parameters } & \multicolumn{2}{c}{$\begin{array}{c}\text { First } \\
\text { iteration }\end{array}$} & \multicolumn{2}{c}{$\begin{array}{c}\text { Second } \\
\text { iteration }\end{array}$} & \multicolumn{2}{c}{$\begin{array}{c}\text { Third } \\
\text { iteration }\end{array}$} & \multicolumn{2}{c}{$\begin{array}{c}\text { Fourth } \\
\text { iteration }\end{array}$} \\
\cline { 2 - 8 } & LB & UB & LB & UB & LB & UB & LB & UB \\
\hline CN 2 & -0.25 & 0.30 & -0.40 & 0.10 & -0.40 & -0.02 & -0.30 & -0.10 \\
ALPHA_BF & 0.40 & 1.00 & 0.60 & 1.00 & 0.75 & 1.00 & 0.80 & 0.90 \\
GW_DELAY & 10 & 300 & 35 & 215 & 0 & 133 & 40 & 105 \\
GWQMN & 0 & 2000 & 0 & 1100 & 0 & 715 & 0 & 465 \\
ESCO & 0.80 & 1.00 & 0.87 & 1.00 & 0.91 & 1.00 & 0.94 & 1.00 \\
CH_K & 5 & 130 & 14 & 92 & 0 & 58 & 32 & 81 \\
ALPHA_BNK & 0.00 & 1.00 & 0.45 & 1.00 & 0.43 & 0.85 & 0.63 & 1.00 \\
SOL_AWC & -0.20 & 0.40 & -0.10 & 0.55 & -0.12 & 0.33 & -0.22 & 0.15 \\
SFTMP & -5.00 & 5.00 & -0.20 & 9.00 & 1.00 & 8.30 & -0.30 & 5.90 \\
GW_REVAP & 0.02 & 0.50 & 0.01 & 0.30 & 0.01 & 0.16 & 0.02 & 0.12 \\
RCHRG_DP & 0.00 & 1.00 & 0.00 & 0.50 & 0.00 & 0.30 & 0.00 & 0.16 \\
\hline
\end{tabular}

Table 6 The statistic summary of the simulation results of each iteration using SUFI-2

\begin{tabular}{|c|c|c|c|c|c|c|c|c|}
\hline \multicolumn{2}{|r|}{ Iteration } & $\begin{array}{c}\text { Threshold } \\
\text { value }\end{array}$ & $\begin{array}{c}\text { Behavioral } \\
\text { parameter sets }\end{array}$ & P-factor & R-factor & $\mathbf{R}^{2}$ & NSE & $\mathbf{P} / \mathbf{R}$ \\
\hline \multirow{4}{*}{$\begin{array}{l}\frac{1}{1} \\
\frac{1}{5} \\
-5\end{array}$} & First & 0.40 & 31 & 0.31 & 0.99 & 0.74 & 0.63 & 0.31 \\
\hline & Second & 0.40 & 214 & 0.36 & 0.89 & 0.80 & 0.72 & 0.40 \\
\hline & Third & 0.40 & 741 & 0.56 & 0.92 & 0.79 & 0.76 & 0.61 \\
\hline & Fourth & 0.40 & 1000 & 0.42 & 0.45 & 0.78 & 0.75 & 0.93 \\
\hline Sut & One & 0.60 & 28 & 0.39 & 0.51 & 0.79 & 0.70 & 0.76 \\
\hline
\end{tabular}


- The list of figures

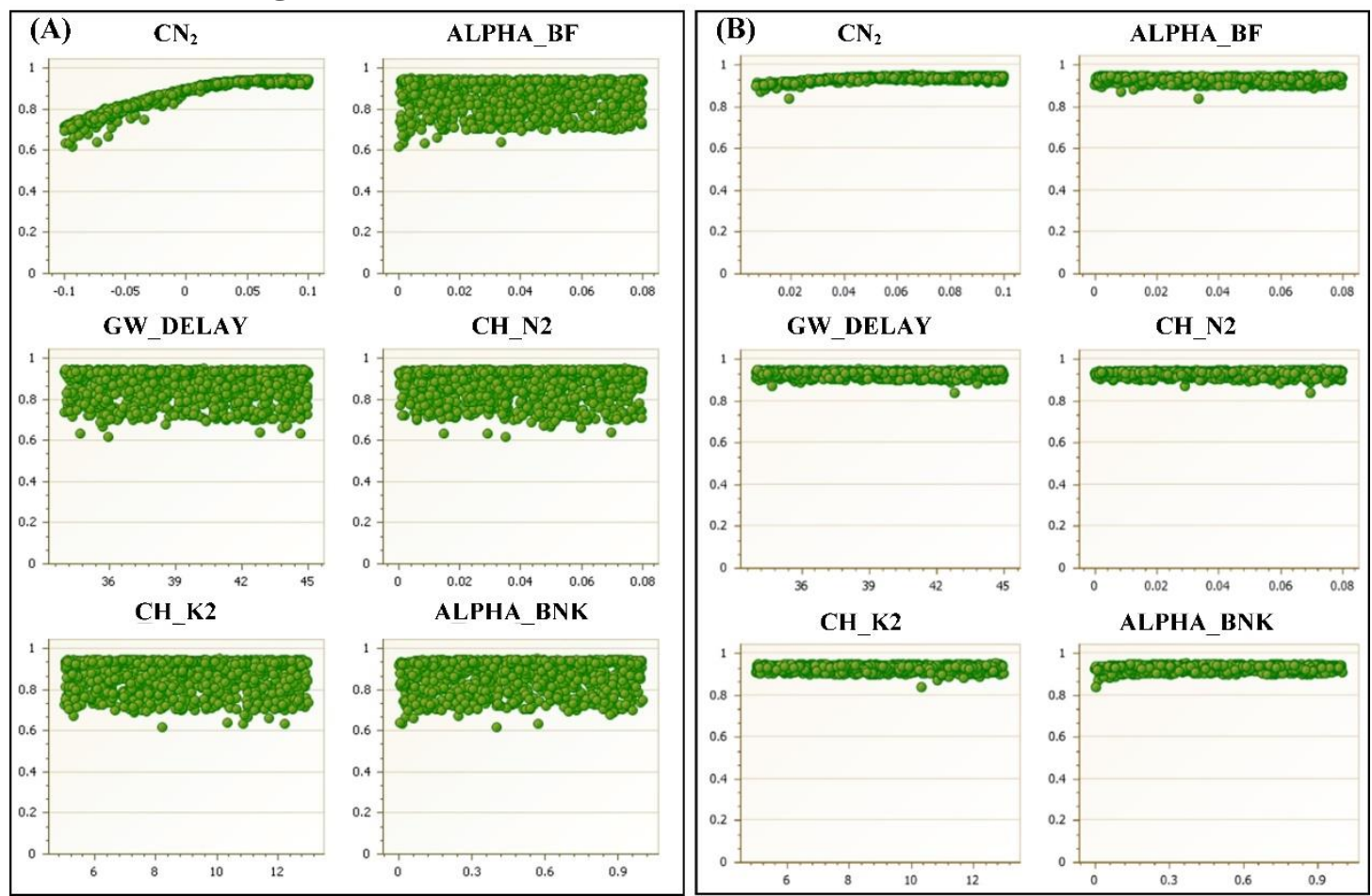

Figure 1 Parameter ranges of selected parameters in the first iteration (A) and second iteration (B)

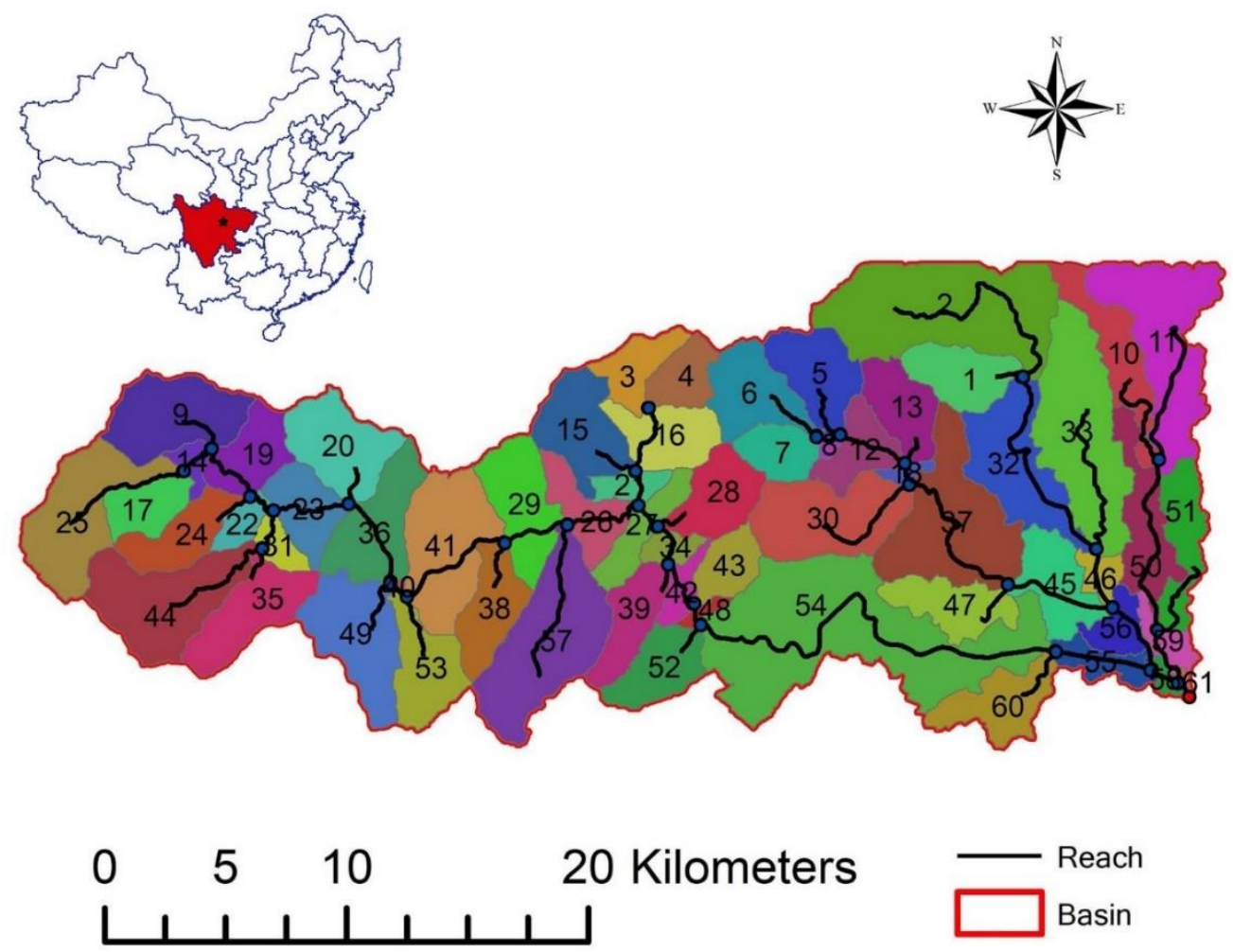

Figure 2 Location map of the upstream of the Wenjing River watershed. The map is generated with ArcMap Version 10.5.(Wu and Chen, 2015) 


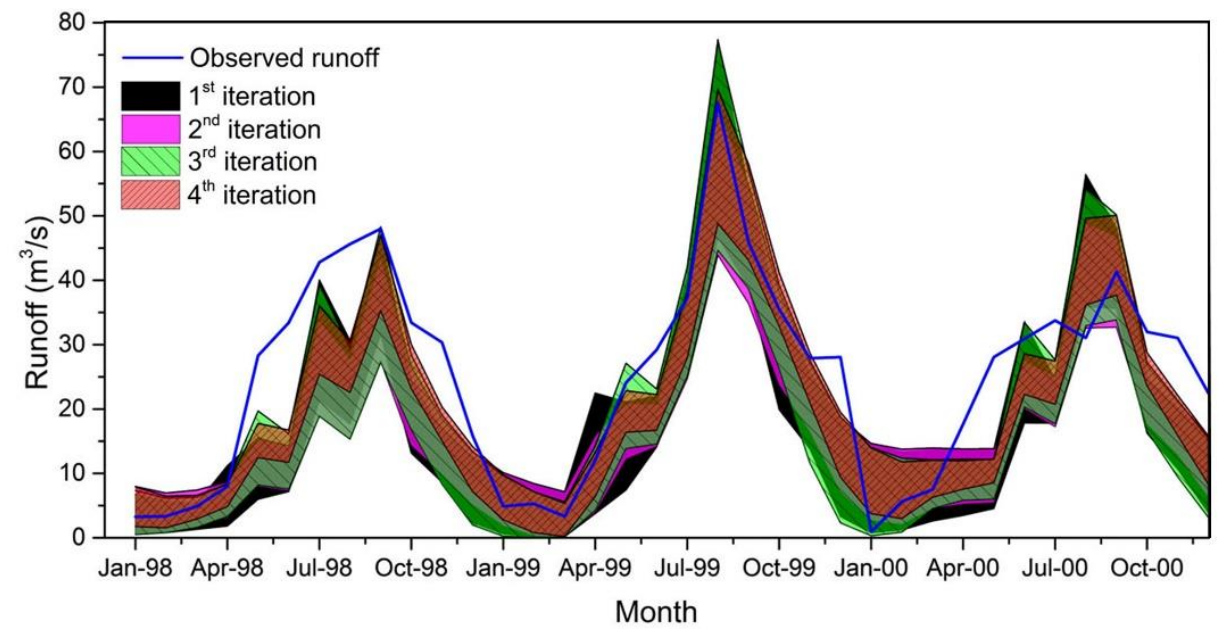

Figure 3 The 95PPU of surface runoff for four iterations using the MS-CUA method

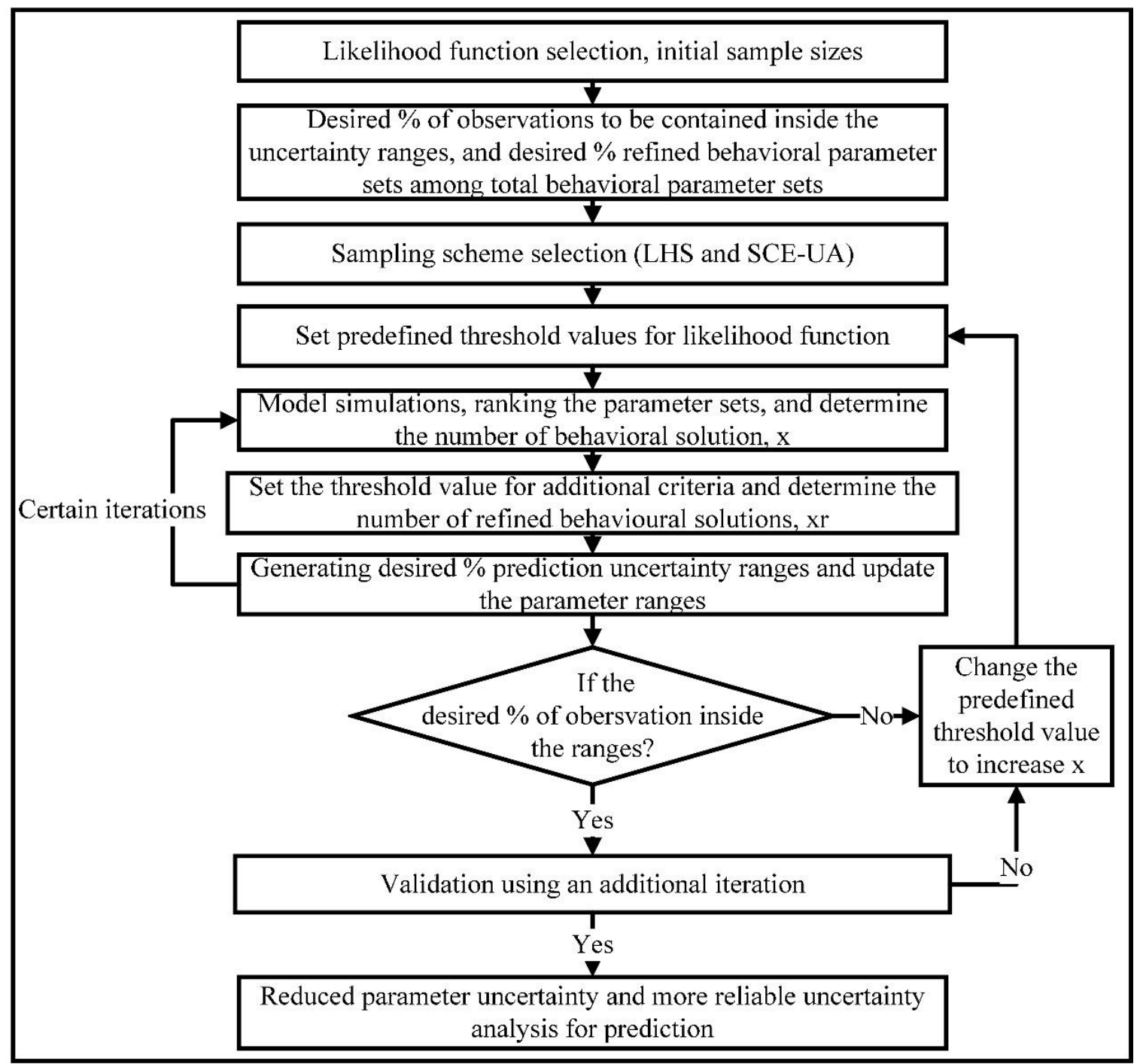

Figure 4. The framework of the MS-CUA method for calibration and uncertainty 
Figures
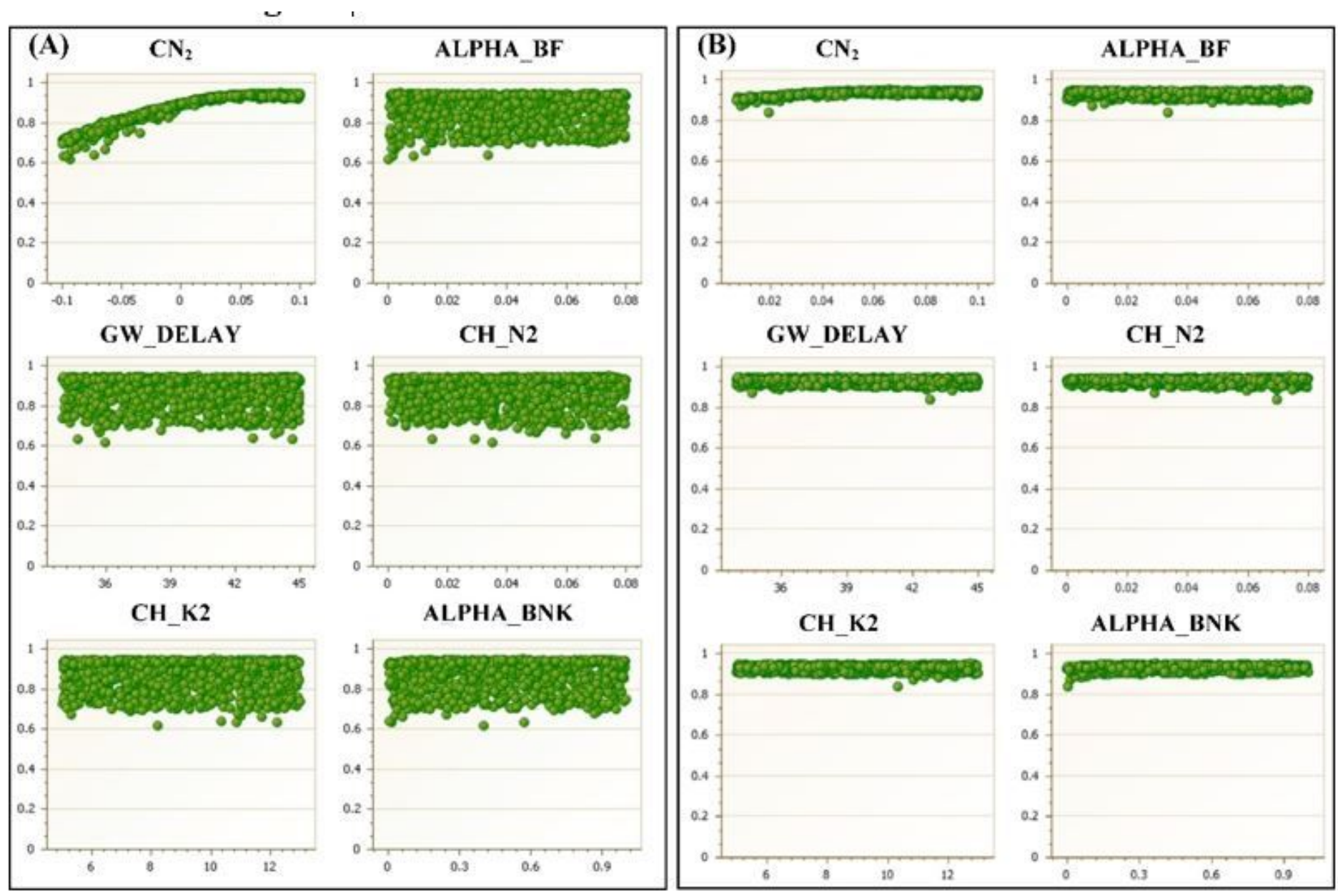

Figure 1

Parameter ranges of selected parameters in the first iteration $(A)$ and second iteration (B) 


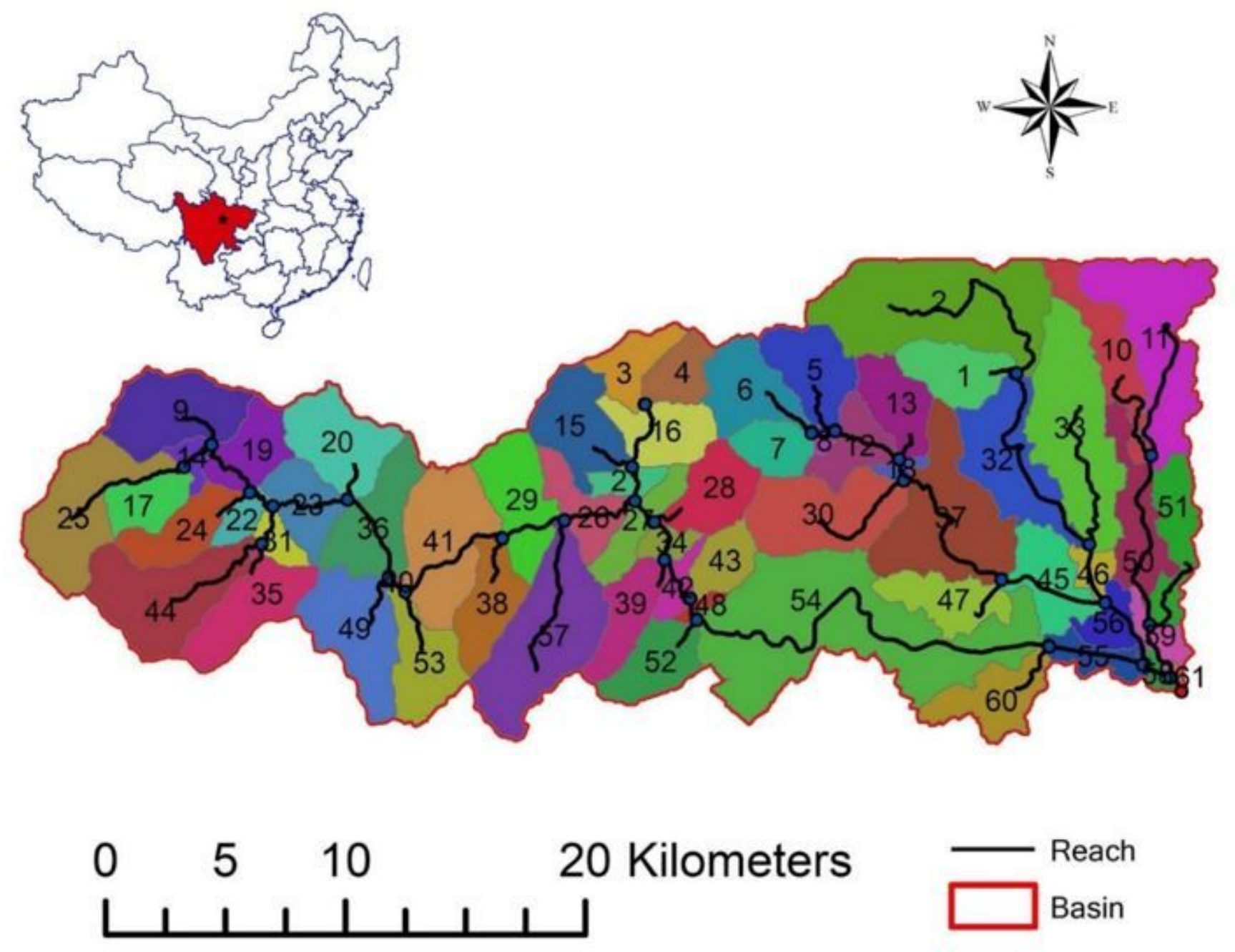

Figure 2

Location map of the upstream of the Wenjing River watershed. The map is generated with ArcMap Version 10.5.(Wu and Chen, 2015) Note: The designations employed and the presentation of the material on this map do not imply the expression of any opinion whatsoever on the part of Research Square concerning the legal status of any country, territory, city or area or of its authorities, or concerning the delimitation of its frontiers or boundaries. This map has been provided by the authors. 


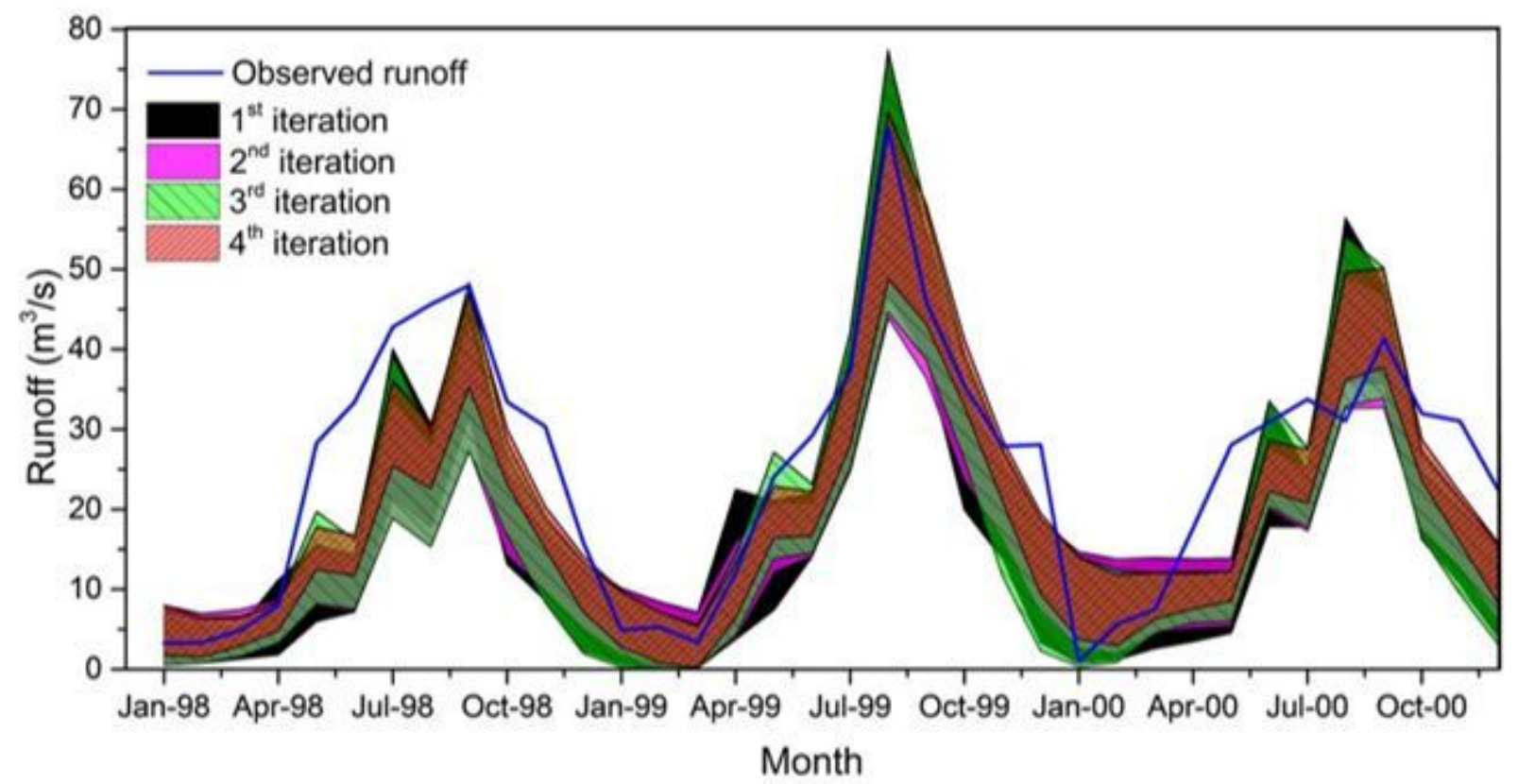

Figure 3

The 95PPU of surface runoff for four iterations using the MS-CUA method 


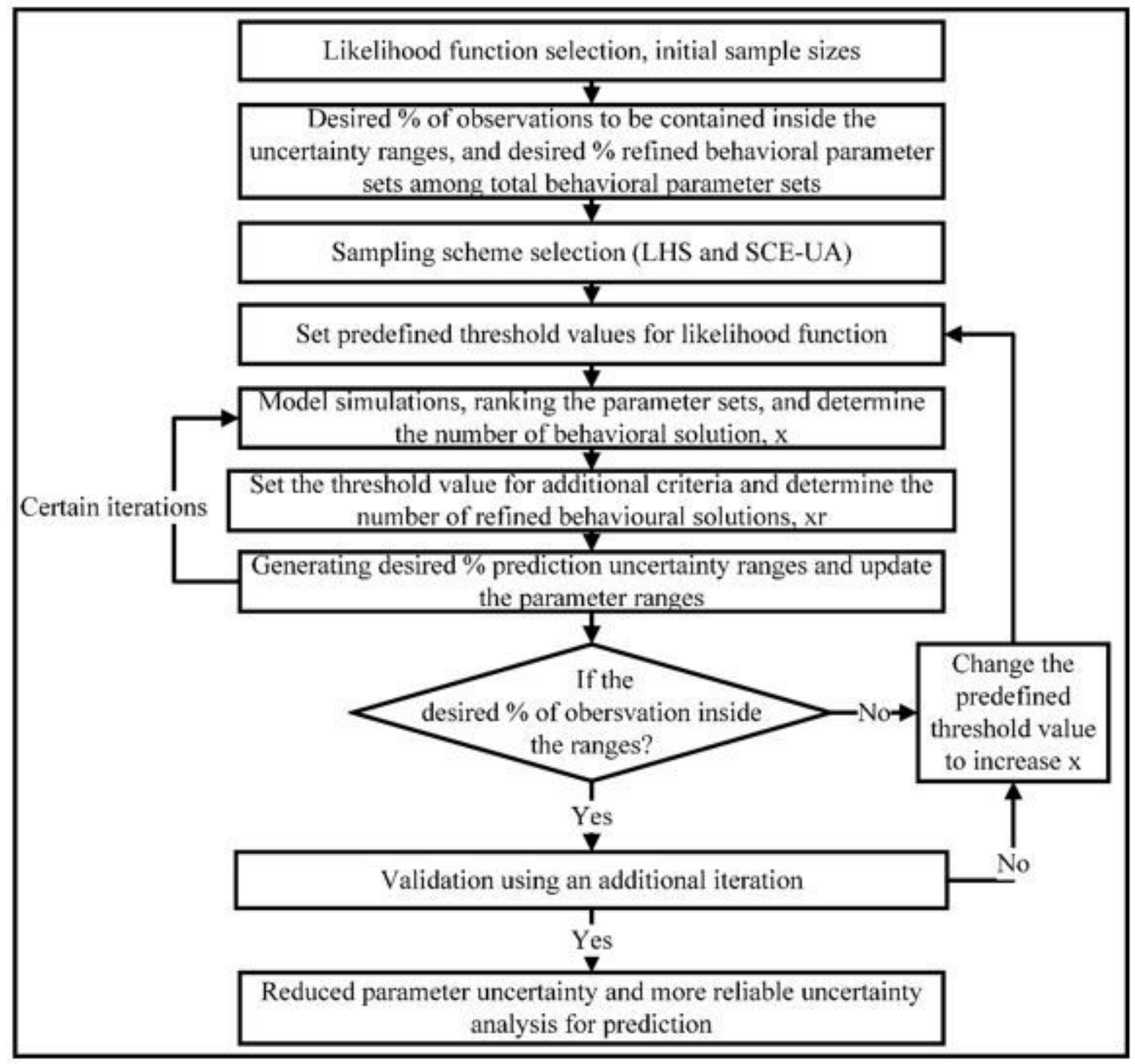

Figure 4

The framework of the MS-CUA method for calibration and uncertainty 